Int. J. Electrochem. Sci., 15 (2020) $8552-8562$

\title{
Simultaneous Determination of Three Nitroaniline Isomers by $\beta$-cyclodextrins ( $\beta$-CDs) and Graphene Quantum Dots (GQDs) Composite Modified Glassy Carbon Electrodes
}

\author{
Shuqing Dong ${ }^{1}$, Qing Bi ${ }^{3}$, Jianyu Qiao ${ }^{1,2}$, Shijun Shao ${ }^{1}$, Xiaoquan Lu ${ }^{3, *}$ \\ ${ }^{1}$ CAS Key laboratory of Chemistry of Northwestern Plant Resources and Key laboratory for Natural \\ Medicine of Gansu Province, Lanzhou Institute of Chemical Physics, The Chinese Academy of \\ Sciences, Lanzhou 730000, PR China \\ ${ }^{2}$ University of Chinese Academy of Sciences, Chinese Academy of Sciences, Beijing 100049, PR \\ China \\ ${ }^{3}$ Key Laboratory of Bioelectrochemistry \& Environmental Analysis of Gansu Province, College of \\ Chemistry \& Chemical Engineering, Northwest Normal University, Lanzhou 730070, China \\ *E-mail: luxq@nwnu.edu.cn
}

doi: $10.20964 / 2020.09 .20$

Received: 8 October 2019 / Accepted: 25 December 2019 / Published: 10 August 2020

\begin{abstract}
Nitroaniline isomers as toxic and environmental pollutant, their detection and recognition is an important task. A novel electrochemical sensor based on $\beta$-cyclodextrins ( $\beta$-CDs) and graphene quantum dots (GQDs) composite modified glassy carbon electrodes ( $\beta$-CDs-GQDs/GCE) has been fabricated for detection and discrimination of three nitroaniline isomers simultaneously. The $\beta$-CDs-GQDs/GCE electrode were characterized by different electrochemical techniques. The three isomers exhibited obvious peak current and potential differences on the modified electrode by cyclic voltammetry (CV) and differential pulse voltammetry (DPV). Because of the selective formation of H-bonds between $\beta$ CDs-GQDs composites and three nitroaniline isomers, the electrochemical recognitions were achieved. Especially, this recognition method relies on not only the peak currents but also the different oxidation potentials. Furthermore, the satisfactory results were obtained for the three noxious nitroaniline isomers simultaneous detection in two pesticides practical samples, when this electrode was applied. The investigates results show that $\beta$-CDs and GQDs are suitable material for isomers sensor. Moreover, its applicability in environmental contaminants promises its great potential utility in the near future.
\end{abstract}

Keywords: Nitroaniline isomers, $\beta$-CDs-GQDs/GCE, Electrochemical recognition, Environmental Contaminants.

\section{$\underline{\text { FULL TEXT }}$}


(C) 2020 The Authors. Published by ESG (www.electrochemsci.org). This article is an open access article distributed under the terms and conditions of the Creative Commons Attribution license (http://creativecommons.org/licenses/by/4.0/). 